\title{
Information in Reality: Logic and Metaphysics
}

\author{
Joseph E. Brenner
}

joe.brenner@bluewin.ch, Chemin du Collège, Les Diablerets, Switzerland

\begin{abstract}
The recent history of information theory and science shows a trend in emphasis from quantitative measures to qualitative characterizations. In parallel, aspects of information are being developed, for example by Pedro Marijuan, Wolfgang Hofkirchner and others that are extending the notion of qualitative, non-computational information in the biological and cognitive domain to include meaning and function.

However, there is as yet no consensus on whether a single acceptable definition or theory of the concept of information is possible, leading to many attempts to view it as a complex, a notion with varied meanings or a group of different entities. In my opinion, the difficulties in developing a Unified Theory of Information (UTI) that would include its qualitative and quantitative aspects and their relation to meaning are a consequence of implicit or explicit reliance on the principles of standard, truth-functional bivalent or multivalent logics. In reality, information processes, like those of time, change and human consciousness, are contradictory: they are regular and irregular; consistent and inconsistent; continuous and discontinuous. Since the indicated logics cannot accept real contradictions, they have been incapable of describing the multiple but interrelated characteristics of information.

The framework for the discussion of information in this paper will be the new extension of logic to real complex processes that I have made, Logic in Reality (LIR), which is grounded in the dualities and self-dualities of quantum physics and cosmology. LIR provides, among other things, new interpretations of the most fundamental metaphysical questions present in discussions of information at physical, biological and cognitive levels of reality including, especially, those of time, continuity vs. discontinuity, and change, both physical and epistemological. I show that LIR can constitute a novel and general approach to the non-binary properties of information, including meaning and value. These properties subsume the notion of semantic information as well-formed, meaningful and truthful data as proposed most recently by Luciano Floridi. LIR supports the concept of 'biotic' information of Stuart Kauffmann, Robert Logan and their colleagues and that of meaningful information developed by Christophe Menant.

Logic in Reality does not pretend to the level of rigor of an experimental or mathematical theory. It is proposed as a methodology to assist in achieving a minimum scientific legitimacy for a qualitative theory of information. My hope is that by seeing information, meaning and knowledge as dynamic processes, evolving according to logical rules in my extended sense of logic, some of the on-going issues on the nature and function of information may be clarified.
\end{abstract}

Keywords: logic, reality, contradiction, dynamic opposition, process, levels, qualitative, meaning.

Despite the widely varying content of theories of information, their emphasis has been on the quantitative aspects of information and their mathematical, abstract and essentially passive character, although information frequently involves human agents as active senders and receivers (Van Benthem \& Van Rooy, 2003).This paper, however, focuses on the qualitative, causal properties of information. The framework for discussion will be my new extension of logic to real complex processes, Logic in Reality (LIR) (Brenner, 2008). In my view, information is a phenomenon which, like human consciousness and change, instantiates real contradictions. LIR, in contrast to standard logics, is capable of describing such contradictions in physical, biological and cognitive processes, permitting stable inferences about them.

The next Section 1 indicates some current approaches to the definition of information and of a unified theory of information. I proceed in Section 2 with an overview of LIR as a complete but nonstandard logic, including its categorial ontology. In Section 3, I will propose an LIR philosophy of information, without pretending that it is a complete or unified theory. In Sections 4-7, different concepts of information are analyzed from the LIR perspective. 


\section{Recent Developments and Directions}

Some examples of recent developments, related to LIR, are as follows: Pedro Marijuan (2009), Wolfgang Hofkirchner and others in the Foundation of Information Science (FIS) initiative are extending the notion of qualitative, non-computational information in the biological (Kaufman et al., 2007) and cognitive domain and include meaning (Menant, 2011) and function. Marijuan (2010) suggests that rather than the outcome of a single, particularized conceptual discussion, "information becomes the intellectual adventure of developing a 'vertical' or 'transdisciplinary' science connecting the different threads and scales of informational processes, which demands both a unifying and multi-perspective approach".

The recent history of information theory and science shows increased interest in qualitative characterizations. Mark Burgin's General Information Theory (2003) refers to a qualitative theory of information as one of its sub-theories. The evolution of current concepts of information has been well summarized by Robert Logan in his What Is Information? (forthcoming). Earlier, Rafael Capurro (2003) asked a similar question in developing a hermeneutical alternative to the standard paradigms of information science. Sören Brier (2008) explains why "information is not enough".

The qualitative properties of information suffer from being viewed as imprecise, inconsistent, subjective, value-laden and context-dependent, rendering rigorous discussion and progress difficult. Semantic and algorithmic approaches to the quantitative aspects of information are mathematically tractable, while the qualitative cannot be subsumed under the standard logical criteria of bi- or multi-valent truth-functionality. Logic in Reality can improve this situation by addressing issues of qualitative information and giving them proper ontological value.

\section{Logic in Reality (LIR)}

\subsection{Axioms, Calculus, Semantics}

Logic in Reality (LIR) is a new kind of logic that extends its domain to real processes, relating them to an underlying particle/field view of the universe. Its axioms and rules provide a framework for explaining the evolution of real world entities and processes at all levels of reality and complexity.

The term Logic in Reality (LIR) is intended to imply both 1) that the principle of change according to which reality operates is a logic embedded in it, the logic in reality; and 2) that what logic really is or should be involves this same real physical-metaphysical but also logical principle.

Details of LIR are provided in Brenner (2008), but its most important axioms are that 1) every real complex process is accompanied, logically and functionally, by its opposite or contradiction (Principle of Dynamic Opposition), but only in the sense that when one element is (predominantly) present or actualized, the other is (predominantly) absent or potentialized, alternately and reciprocally, without either ever going to zero; and 2) the emergence of a new entity at a higher level of reality or complexity can take place at the point of equilibrium or maximum interaction between the two.

LIR is a logic applying to interactive processes, in a process-ontological view of reality, to trends and tendencies, rather than to 'objects' or the steps in a state-transition picture of change. Processes are described formally as transfinite chains of chains of chains, etc. of alternating actualizations and potentializations of implications, considered with the other logical operators, conjunction and disjunction as real processes themselves. The directions of change are either 1) toward stable macrophysical objects and simple situations, the result of processes of processes, etc. going in the direction of a "non-contradictory" identity or diversity: or 2) toward a state of maximum contradiction (T-state for included third term) from which new entities can emerge. LIR is, therefore, a logic of emergence, a new non-propositional, non-truth-functional logic of change.

Standard logic underlies, rather, the construction of simplified models which fail to capture the essential dynamics of biological and cognitive processes, such as reasoning (Magnani, 2002). LIR does not replace classical binary or multi-valued logics but reduces to them for simple systems and 
situations. The interactive relationships within or between levels of reality to which LIR applies are characteristic of complex entities with some form of internal representation, biological or cognitive.

The levels of reality referred to in LIR are ontological, defined by the different, but isomorphic physical laws that apply. The LIR view of reality as constituted by levels can be compared to Floridi's Levels of Organization (forthcoming) which also support an ontological approach, and contrasted with his epistemological Levels of Abstraction.

\subsection{The Categorial Ontology of LIR: Inconsistency}

In the categorial ontology of LIR, the sole material category is Energy, and the most important formal categories are Process and Dynamic Opposition, and the sub-categories of Separability and Non-Separability. This is the critical categorial feature of the LIR process ontology, the NonSeparability of opposing phenomena, e.g., two theories or elements of phenomena, e.g., syntax and semantics, types and tokens.

From the LIR metaphysical standpoint, for real systems or phenomena or processes in which real dualities are instantiated, their terms are not separated or separable! Real complex phenomena display a contradictional relation to or interaction between themselves and their opposites or contradictions. Note that the requirements in classical 1) category theory of exclusivity and exhaustivity and 2) set theory of absolute separation of sets and their elements do not apply: they are bivalent logic in another form.

LIR thus approaches in a new way the inevitable problems resulting from the classical philosophical dichotomies as well as such concepts as space and time, or simultaneity and succession as categories with separable categorial features. Non-Separability underlies all other metaphysical and phenomenal dualities, such as cause and effect, determinism and indeterminism, subject and object, continuity and discontinuity, and so on. I thus claim that Non-Separability at the macroscopic level, like that being explored at the quantum level, provides a principle of organization or structure in macroscopic phenomena that has been neglected in science and philosophy.

In contrast to standard logics, LIR has no difficulty in dealing with inconsistency, interpreting it as a natural consequence of the underlying principle of dynamic opposition in physical reality. Resolution of the (endless) debate about the nature of change (Mortensen, 2008), seems to require a fundamental inconsistency in the world, which LIR naturalizes.

\subsection{LIR and Other Logics}

LIR resembles paraconsistent logics (PCL), in which the law of non-contradiction fails. Paraconsistent logics (PCL) are defined such that contradiction does not entail triviality (Carnielli, 2005). According to the LIR axiom of Conditional Contradiction, however, if $A$ and non-A are present at the same time, it is only in the sense that when $A$ is (predominantly) actual, non- $A$ is (predominantly) potential.

The Universal Logic of Huacan He (2005) and Jean-Yves Béziau includes and can explicate all standard logics. LIR, however, falls outside its scope, due to its non-propositional, non-standard probability-like metavariables which, as in quantum logics do not follow the laws of commutation or distribution.

\subsection{Information Logic}

A first step toward developing a logic applicable to information has been made by Floridi (2006); his Information Logic (IL), or Logic of Being Informed, recognizes something static and abstract about standard formulations of doxastic logics that depend on the (tripartite) notion of knowledge as justified true belief. Logic in Reality ascribes a logical, non-metaphorical content to descriptions of an antagonistic interaction between the individual and the world, as an on-going process (Brenner, 2009). It is an informational process in which both actors change as the reactions of one or the other, alternately, predominate. Rather than a Logic of Being Informed, LIR is a Logic of Informing. 


\subsection{Cognitive Aspects of LIR}

The LIR epistemological approach to consciousness analyzes the details of the acquisition of perceptions, and postulates that afferent stimuli are 'split' into conscious potentialities and unconscious actualities. Higher levels of cognition develop from subsequent interactions with both internal and externally related (efferent) processes. Thus while your mind is physically external to mine, some of its perceptible potentialities can be internalized by me, perhaps by mirror neurons in the concept of Ramachandran. This principle applies to the interaction between the sender and receiver of information, in the usual model, placing them on the same basis. LIR thus provides a logical foundation for discussion of ethical questions based on kinds of information that complements IL. Both are reconsiderations of logic that, as Marijuan suggests (2009), may be necessary for the advancement of information technology in an ethical direction (Brenner, 2009).

\section{The LIR Approach to Information}

\subsection{The Components of Information}

Based on the contradictorial principles of LIR outlined above, what information is in reality and what constitutes a proper theory of information, of which information is its substrate, cannot be totally separated. Further, the real properties of informational entities or processes, binary and non-binary, are not independent of and cannot be discussed without reference to the a priori non-binary energetic processes that are their source, in some real situation, at all levels of reality.

The LIR approach thus incorporates and provides for a relation between two complementary components of information: 1. information as well-formed, meaningful and truthful data (Floridi, forthcoming); and 2. information as real energetic processes, whereby information-as-processes can function as higher-level operators on information-as-data at a lower level of reality.

\subsection{The Floridi Perspectives and LIR}

In Floridi's Philosophy and Logic of Information, information can be viewed from three perspectives: information as reality (e.g., as patterns of physical signals, which are neither true nor false), also known as environmental information; information about reality (semantic information, aletheically qualifiable); and information for reality (instructions, like genetic information, algorithms, etc.).

Extensionalist approaches to the definition of information as reality or about reality provide different starting points for answering the question of what information is. The approaches listed in (Floridi, forthcoming) that are most relevant to Logic in Reality are defined as 3. probabilistic, 4. modal, 5. systemic, 6. inferential and 7. semantic.

I feel that a firm distinction cannot be maintained between these different approaches insofar as information as reality is concerned. Thus, if information describes real process systems and these in turn are described as probability distributions, they share aspects of 3,4 and 5 . To the extent real information processes are inconsistent, 4 is required, and if the description is a logical one (in the extended sense of logic of Logic in Reality, LIR), inference and therefore 6 is also involved.

This leaves definition 7 but let us assume that we are at the lowest level or reality. LIR states that there is no absolute disjunction between this level of reality and those to which the more complex concepts of information apply. Logic in Reality provides 1) a physical and logical grounding for a real, dialectical interaction between informational levels, such that information at any level shares some of the properties to some extent of the structure of the information at the levels above and below it; and 2) a focus on information that is complex and value-laden which, unlike simpler data, is not and does not have to be decoupled from its support.

The basic concept of information in the LIR logic of processes, namely that logical (in the LIR sense) information is the actual state of the world. Information in LIR includes, but is not limited by, the standard characteristics of information.

A definition of information that is most congenial to LIR was made by Kolmogorov (Mindell \& Gerovitch, 2003), namely, that information is any operator which changes the distribution of prob- 
abilities in a given set of events. This is quite different from his well-known contribution to algorithmic information theory, but fits the process conceptions of LIR.

In LIR, where logical elements of real processes resemble (non-Kolmogorovian) probabilities, the logical operators are also processes, such that a predominantly actualized positive implication, for example, is always accompanied by a predominantly potentialized negative implication.

LIR can thus provide bridging concepts or 'glue' between semantic information at the lowest informational level and higher ones. It is not a new concept that higher levels of information subsume aspects of semantic information. What LIR does is to place the concept of information in a naturalized physical, metaphysical and logical context. Information is thus both a means to model the world and part of the world that is modeled, and LIR describes the dialectic relation between them.

\section{Toward a Unified Theory of Information}

Hofkirchner's (2009) approach to a Unified Theory of Information (UTI) is to eliminate the absolute and in my view artificial separation between critical concepts of information in favor of a dialectical relationship similar to the ancient intuition of 'unity-in-diversity'. Specifically, his "UTI seeks a concrete-universal concept of information rather than an abstract one".

Hofkirchner considers information as a "superconcept", which includes a group of overlapping concepts such as message, signal, etc. as they apply to communication, cognition and cooperation between human and non-human organisms. Hofkirchner asks how matter and idea, mind, information, etc. can be grasped as complements and with them information as a thing (a structure, a flow) or as a human construction. Hofkirchner gives a dialectical answer to the implied division between subject and object, suggesting that mind, and with it information, is of a different 'materiality' than 'non-emergent' states of matter.

From the LIR standpoint, mind and information can be seen as "complements" if ones sees them as processes. Structure, flow and "human processing activity" all follow the same real, physical dialectics. If matter and information are differentiated in a "common genus", for LIR, that genus is simply energy, and both follow its logical patterns of evolution, avoiding the problems of the term "different materiality". Logic in Reality is, also, a logic of emergence or "emergent materialism". In this view, information is, pace Wiener, an energetic phenomenon that instantiates real contradictions.

Hofkirchner wishes to avoid reliance on a "formal-logical figure of necessary and sufficient conditions" and use a way of thinking that integrates as well as differentiates the particular and universal", with which LIR agrees. LIR is grounded in the fundamental physical dualities of the universe, and provides a principled basis for such contradictorial relations in real emergent physical processes. These no longer constitute the standard formal-logical figures that Hofkirchner correctly critiques, but a dynamic logic of information-with-meaning that could support a UTI.

\section{Qualitative Information Theory}

Burgin's new General Theory of Information (GTI) (2003) treats information from a pragmatic, dynamic perspective, involving changes of structure or behavior of the receiver (and, as below, of the sender as well). While Kolmogorov (1965), following Shannon, discussed several approaches to defining the notion of the amount of information, the work of Marian Mazur and Burgin's own view of a GTI refers to a qualitative theory of information as one of its sub-theories.

Burgin's definition of Qualitative Information Theory is "information (that) is a transformation of one communication of an information association into another communication of the same association", using some realistic information measure. This concept in my view is necessary but not sufficient to capture the all the qualitative properties of information, its positive or negative "valence" that can be dependent or independent of the sender's intentions, but can have a differential impact on the receiver.

LIR supports dynamic theories of information, included in this GTI, where information is an action, that is, a process or operator, causing some form of transformation. Marijuan proposes the 
concept of "Information Proceeding" to capture the process by which information always changes the subject and there is "no such thing as a separate observer in the information realm". Logan (forthcoming) also points out the lack of attention paid to the qualitative as opposed to the quantitative aspects of information, that is, the need to incorporate a functional notion of meaning, recalling pioneering work by Donald MacKay.

\subsection{The Valence of Information}

My thesis is that the values conveyed by information should be viewed as positive or negative in terms of their effect on both sender and receiver, and that accordingly the essential quality of information is its intentional valence, positive or negative. This corresponds to a property or characteristic of qualitative information in process terms as a reality in a physical space (as opposed to a data space, cf. Floridi, 2004), in a morally valued interaction between producer and receiver. LIR is neither topic-neutral nor context independent, and can support a view of information involving apparently contradictory perspectives and assigns equal ontological value to negative as well as positive information.

In his discussion of the typological neutrality of information, Floridi defines secondary data "as the converse of primary data, constituted by their absence (one could call them anti-data).... This is a peculiarity of information: its absence may also be informative." The absence of a response to a computer query is also given as a bona fide instance of negative information-as-data. Floridi discusses (Floridi, forthcoming and elsewhere) why, in any truth-functional propositional theory of information, false information and misinformation should not be considered as information at all. But in LIR, we are not dealing with truth-functionality, and negative qualitative information is not falsehood. LIR uses the term negative information to mean intended messages in a necessarily social context that have negative or unnecessarily and unfounded pessimistic content with, probably, negative consequences for the receiver. This point is made by Capurro (2003), who also calls attention to the philosophical necessity, for a theory of information in reality that refers to the existentiality of our "being-in-the-world-with others", to include a discussion of misinformation and its interwovenness (non-separability) from information. In his view, it is the absence of separation that insures that information science is a hermeneutic science and accordingly a foundation of an ethics of information.

There is a further and more 'positive' side to 'negative' information. Marijuan (1996) sees absences, needs, voids, etc. as necessary to provide a complete picture of the infosphere. This concept goes back to the views of McLuhan of "negativity" and the subtle economy involved in predominantly signaling by "absences".

\section{Semiotic Information Theory}

At first sight, the semiotic approach to information might appear to capture its multiple facets, ordering them into the functional categories proposed by $\mathrm{C}$. S. Peirce. Brier has provided a complete current interpretation of Peirce (Brier, 2008).

However, I consider Peirce's theory insufficiently dynamic because there is no energy that can be assigned to his triadic relations that would give them a basis in reality (physics). I see the same problem (Brenner, 2008) with Peirce's categories as with the Hegelian triad of thesis, antithesis and synthesis: there is no deductive basis for the movement from one term to the other or a description of any physical interaction between them. If the argument is made that nothing of the sort is required, my response is that is exactly the problem - the terms are not physically grounded and hence have limited explanatory value other than as a heuristic device for keeping track of the entities involved in biological processes; its use should not make one neglect the real properties of the system.

The Peircean semiotic concept of information has been summarized by Quieroz, Emmeche, and El-Hani (2008) (QEE) as a "triadic dependent" process where a form is communicated from an Object to an Interpretant through the mediation of a Sign. My critique of this approach is that as 
stated by Peirce himself, it is derived from a formal science of signs that provides an analytical framework. Thus the QEE approach to information as process is constrained by the abstract characteristics of the Peircean categories, that is, their abstraction from dynamic aspects of real physical phenomena.

In contrast to QEE, I derive the triadic characteristics from the LIR view of the contradictorial evolution of all real processes, providing the physical basis for the QEE differentiation of potential and effective (actual) semiosis and consequent definition of potential and effective information as well. In LIR, information is a complex of processual interactions with both binary (dyadic) and ternary (triadic) properties, all of which can be predominantly actualized (effective) or potentialized (not effective) at any time. This would seem preferable to the nebulous concept of a Sign as a Medium for communication of Form.

The essentially static linguistic definition of Form in terms of "conditional propositions" states that certain things would happen under certain circumstances. Strikingly, as quoted by QEE, Peirce said that "Form can also be defined as potentiality ('real potential': EP 2.388) (emphasis mine). In LIR, structure and form are also physical processes, including the physical processes of their conceptualizations. Form is characterized not as 'potential' only, but as a process whose elements are both actual and potential at the same time.

LIR confirms the QEE critique of the argument by Jablonka that "for a source to be an information input rather than merely a source of energy or material, its form, or variations in its form, rather than any other attribute should affect the interpreter's response in a consistent, regular way". Here, a distinction has been created according to which form is idealized as something non-energetic, but still with causal properties. To say that form is also energy is not to make a physicalist reduction, but to avoid conceptualizing it out of existence.

\section{Biological Information Theory and Meaning}

\subsection{Meaning in LIR}

Like information, meaning has many definitions, which run from "something that is signified, especially by language" to "something that is felt to be the inner significance of something". I see in this polysemy the same conceptual range as from standard logic to LIR, that is, from a method for managing linguistic phenomena to a description and explanation of the structure of the world.

In the LIR dynamic view of meaning, it is what is felt that is significant, in other words, meaningas-experience, and my experience of the world. Everything logical in the LIR sense is experimental or existential, has a meaning and is meaning because it not only emerges from the underlying dualities of energy, but is directed toward the relationship with the other - 'meaning for'. Meaning is thus inherent to all dynamic processes.

In the LIR interactionist picture, meaning, like consciousness, is a necessary consequence of the existence of the physical world and its characteristics. As Peruzzi (1994) puts it, it is the furniture of the base macro-world (light, chemical bonds, gravity, temperature) that molds conditions on the earth's surface and living beings, among other things for the emergence of language. Language cannot be separated from its basis in perception, and the "Scylla of relativism and the Charybdis of idealism" can be avoided by maintaining the flux of meaning from perception and action to cognition. The consequence, that conforms to the LIR category of Non-Separability, is that there cannot be any cut between perception and cognition without depriving sentences of meaning. The properties of LIR are not arbitrary. Both the structure of objects and the patterns of perceptual interaction with objects establish features of both LIR and language.

\subsection{Biotic Information}

In their key paper (Kaufmann et al., 2007), Kauffman, Logan and their colleagues propose a new reading of information inherent in biological processes that unite matter, energy and information that is compatible with and supported by Logic in Reality. They show that neither the Shannon definition of information as a scalar quantity of bits, devoid of meaning, nor Kolmogorovian informa- 
tion which refers to standard probability distributions of non-interactive systems, is applicable in biology. Information should be designated as 'instructional' or 'biotic' in the sense that it carries meaning and consists of constraints or their physical equivalents - boundary conditions that also partially cause events. Most importantly, the coming into existence of the constraint is itself part of the propagating organization of the entity. "Constraints are information and information is constraints." This recursive aspect is characteristic of non-Markov chains, the non-Kolmogorovian probability behavior of two mutually dependent entities to which LIR applies.

LIR proposes a "missing ingredient" of dynamic opposition or antagonism that reinforces this picture of information for the evolution of living systems, without violating any principle of physical closure. It provides a cybernetic explanation of how constraints-as-information in their physical manifestations can be causally effective when identified with the residual potentialities of all material structures more complex than an isolated quantum particle. By locating the causal powers of constraints in the physical potentials available "from the bottom up", one has at least part of an explanation of why structures, up to and including social structures, have the properties they have. (In this connection, one can introduce the term 'constraining' in addition to constraint, the participle giving a more accurate description of a process.)

Christophe Menant (2011) looks at information in the relation between an arbitrary real signal and an entity or system capable of interpreting that signal. In this "bottom-up" explanation of information and meaning, a meaning is meaningful information generated by some purpose or action in connection with a constraint on that system. "The generated meaning is precisely the connection (I would add the emergent connection) between the received information and the constraint." That the meaning associated with an outside entity depends on the entity and also on the internal state of the system I see as a logical process that follows the rules of LIR of non-separability and alternating actualization and potentialization.

LIR confirms the views of Brooks, cited by Menant that "It turns out to be better to use the world as its own model". I propose the LIR principle (Lupasco, 1986), free of any vitalist flavor, as a candidate for the "something fundamental and currently unimagined in our models of biology" that Brooks thinks we might be missing.

\subsection{Biological Information Theory: The Mirage of Self-Organization}

Any theory of biological development or becoming must capture the duality of biological systems, that is, the composition of living systems by non-living substrates. The LIR dynamic process ontology is grounded in the contradictorial dualities of physics and the coexistence of actuality and potentiality in all real systems. Its principles have the advantage of providing an explanation for ontic, non-epistemological emergence of more complex entities, including living systems, from less complex substrates based on the residual, physical potentialities of all particles above the level of quanta, that is, protons, neutrons, and the hydrogen atom, without recourse to arbitrary notions of self-organization or auto-catalysis. In our world, the initial pre-biotic conditions are not completely random.

From the LIR perspective, there is no need to postulate totally autonomous agents (Riofrio, 2011) or real-world systems (Hofkirchner, 2011) capable of "spontaneous self-organization". This strategy only begs the question of the origin of the capacities for that "self"-organization. The wellknown chain of argument, from Prigogine through Varela to Kauffmann is not totally incorrect, but it is incomplete and partially misleading. "Matter-energy variations" have an effect on biological processes, but not as Peircean signs: they are, already, information-with-meaning.

\section{Conclusion and Outlook}

In this paper, I have discussed information in terms of a new kind of logic, Logic in Reality (LIR). LIR is an extension of logic to real phenomena, grounded in quantum mechanics and applicable to complex real processes. It is a method of analyzing the underlying dynamics of information and information transfer. Unlike standard bivalent or multivalent propositional and predicate logics, even 
in their modal or deontic versions, LIR provides a basis for describing the behavior and evolution of real systems in logical terms, and information itself in the same terms. Similar logical principles, in this view, govern the nature of knowledge and the relation of information and meaning. LIR is sufficient as a part of a description of information, but also necessary since without it the physical driving forces for the behavior of information as a meaning-laden process cannot be properly associated with conceptual epistemological descriptions of information in terms of Levels of Abstraction that are observer-dependent.

Logic in Reality thus provides a new contradictorial description of information as it is in reality. Information is reality in a way that includes higher dimensional cognitive processes relevant to theories of the emerging Information Society and its non-informational components. The development of a proper theory of information is thus an eminently transdisciplinary task, and Hofkirchner, Fuchs, Marijuan and other information scientists have called attention to the transdisciplinary aspects of the ICTs both as such an in relation to society.

The LIR theory of information is not intended to supersede any or all existing approaches. It is proposed as a logical methodology that would encourage the retention and use of partially conflicting notions of information as produced and used. Like standard bivalent and multivalent logic, digital conceptions of information will persist and support the further development of computer science and engineering. From the LIR perspective, such developments are not only probable, but essential to the overall development of the understanding of "information in reality". The Universal Logic of $\mathrm{He}$ (He et al., 2005) describes the mathematical dialectic aspects of logic and LIR the nonmathematical ones may both be needed for a science of information. My hope is that the approach of Logic in Reality, suggested in this paper, may favor a proper balance between information-asdata, and information-as-process, as well as the more complex normative worldview embodied in the latter.

\section{References}

Brenner, J. E. (2008). Logic in reality. Berlin; New York, NY: Springer.

Brenner, J. E. (2009). Prolegomenon to a logic for the Information Society. tripleC, 7(1), 38-73.

Brier, S. (2008). Cybersemiotics: Why Information is not enough. Totonto, CA: University of Toronto Press.

Burgin, M. (2003). Information theory: A multifaceted model of information. Entropy, 5, 146-160.

Capurro, R. (2003). Foundations of information science: Review and perspectives. Retrieved from http://www.capurro.de/tampere91.htm.

Carnielli, W., Marcelo, E. C., \& Marcos, J. (2005). Logics of formal inconsistency. CLE e-Prints, 5(1).

Floridi, L. (2004). Open problems in the philosophy of information. Metaphilosophy. 35(4), 554-582.

Floridi, L. (2006). The logic of being informed. Logique et Analyse, 49(196), 433-460.

Floridi, L. (forthcoming). The philosophy of information. Oxford, UK: Oxford University Press.

$\mathrm{He}, \mathrm{H}$. et al. (2005). Principle of universal logics. Beijing: China Science Press.

Hofkirchner, W. (2009). How to achieve a unified theory of Information. tripleC, 7(2), 357-358.

Hofkirchner, W. (2011). Does computing embrace self-organization? Ontological problems of information-proessing. In G. Dodig-Crnkovic \& G. Burgin (Eds.), Information Computation: Essays on Scientific and Philosophical Understanding of Foundations of Information and Computation. World Scientific Series in Information Studies, vol. 2 (chapter 7). Singapore: World Scientific Publishing.

Kauffman, S., Logan, R., Este, R., Goebel, R., Hobill, D., \& Shmulevich, I. (2007). Propagating organization: An enquiry. Biology and Philosophy, 23, 27-45

Kolmogorov, A. N. (1965). Three approaches to the definition of the quantity of information. Problemy peredachi Informatsii, 1(1), 3-11

Logan, R. K. (forthcoming). What is Information? Toronto, CA: University of Toronto Press.

Lupasco, S. (1986). L'énergie et la matière vivante. Monaco: Le Rocher.

Magnani, L. (2002). Preface to Model Based Reasoning: Science, Technology, Values. Dordrecht, NL: Kluwer.

Marijuan, P. C. (1996). Gloom in the society of enzymes: On the nature of biological information. BioSystems, 38, $163-171$.

Marijuan, P. C. (2009). The advancement of Information Science: Is a new way of thinking necessary? tripleC, 7(2), 369375.

Marijuan, P. C. (2010). What is FIS? What is Information? Retrieved from http://fis.icts.sbg.ac.at. 
Menant, C. (2011). Computation on information, meaning, and representation: An evolutionary approach. In G. DodigCrnkovic \& G. Burgin (Eds.), Information Computation: Essays on Scientific and Philosophical Understanding of Foundations of Information and Computation. World Scientific Series in Information Studies, vol. 2 (chapter 10). Singapore: World Scientific Publishing.

Mindell, D. \& Gerovitch, S. (2003) Cybernetics and information theory in the United States, France, and the Soviet Union. In M. Walker (Ed.), Science and Ideology: A Comparative History (pp. 66-95). London, UK: Routledge.

Mortensen, C. (2008). Change. In E. N. Zalta (Ed.), The Stanford Encyclopedia of Philosophy. Retrieved from http://plato.stanford.edu/archives/fall2008/entries/change/.

Peruzzi, A. (1994). Prolegomena to a theory of kinds. In J. Macnamara \& G. E. Reyes (Eds.), The logical foundations of cognition (chapter 8). Oxford, UK: Oxford University Press.

Quieroz J., Emmeche, C., \& El-Hani, C. N. (2008). A Peircean approach to 'information' and its relationship with Bateson's and Jablonka's ideas. The American Journal of Semiotics, 24(1-3), 75-94.

Riofrio, W. (2011). A molecular dynamic network: Minimal properties and evolutionary implications. In In G. Dodig-Crnkovic \& G. Burgin (Eds.), Information Computation: Essays on Scientific and Philosophical Understanding of Foundations of Information and Computation. World Scientific Series in Information Studies, vol. 2 (chapter 12). Singapore: World Scientific Publishing.

Van Benthem, J \& Van Rooy, R. (2003). Connecting the different faces of information. Journal of Logic Language and Information, 12(4), 375-379.

\section{About the Author}

Joseph E. Brenner

was born in Paris in 1934. After primary and secondary education in New York, he received B.A. and M.S. degrees from the University of Chicago. In 1958, he earned a Ph.D. in Organic Chemistry from the University of Wisconsin. His career was in the chemical industry (Du Pont de Nemours International) in R.\&D. and corporate development. In 1998, he began collaboration with the International Center for Transdisciplinary Research (CIRET) in Paris. His focus has been to make the logical system of the Franco-Romanian thinker Stéphane Lupasco (Bucharest, 1900 - Paris, 1988) accessible to English-language readers. Dr. Brenner is a member of the American Association for the Advancement of Science; the New York Academy of Sciences; and the Swiss Society for Logic and the Philosophy of Science. He recently joined the Editorial Board of tripleC. 